\title{
Consequences of stabilising selection for polygenic variation. II. Any number of loci
}

\author{
Kenneth Mather*†
}

School of Biological Sciences, University of Birmingham, P.O. Box 363, Birmingham B15 2TT, U.K.

In effect, this paper is a continuation of Mather (1987), where it was demonstrated that, depending on the properties of a gene pair, stabilising selection could bring about a stable polymorphism, but would not always do so. The analysis is now extended to any number of loci acting simultaneously. Following the earlier paper, the effects of gene differences on the primary character are assumed to show neither non-allelic interaction nor disturbance due to linkage, but different gene pairs will generally show interaction in their effects on fitness which are assumed to be related to the square of primary phenotypic deviations from the optimum.

It is shown that, in principle, the equilibrium allele frequencies at one locus are affected by the average deviation from the optimum of the three genotypes at each of the other polymorphic loci in the system. Thus stabilising selection acts not on individual loci independently, but on the system as an integrated whole: the response to selection at any one locus is affected by all the other loci and, in its turn, it affects response at all the other loci. The allele frequencies at any one locus will be adjusted by the allele frequencies at all the rest and the mean expression of the primary character will thus come to be at the optimum, subject, of course, to the effects of temporary environmental fluctuations.

Published data from experiments based on the Texas cage population of Drosophila melanogaster agree with the theoretical expectations derived from this analysis.

\section{INTRODUCTION}

An earlier paper (Mather 1987) dealt with the consequences for fitness of a gene difference $(A, a)$ affecting the expression of a quantitative character when stabilising selection was acting in favour of an intermediate optimum expression. The analysis showed that such stabilising selection would lead to a stable polymorphism where the heterozygote departed less from the optimal expression of the character than did both of the homozygotes: but where it did not do so, one or other allele would become fixed either by the direct effect of selection or by the resolution of an unstable equilibrium. Preliminary consideration was also given to the inter-relation of two gene pairs $(A, a$ and $B, b)$ and it was shown that the selection pressure on $A, a$ was independent of that on $B, b$ only when the mean expression of the three $B, b$ genotypes was

\footnotetext{
* We record, with regret, the death of Professor Sir Kenneth Mather, CBE, FRS, on 20th March 1990.

† Reprint requests from $\mathrm{Dr}$ M. J. Kearsey at the above address.
}

at the optimum. The analysis is now extended to the situation where any number of loci is involved and the consequences of their interaction are examined.

The familiar parameters of quantitative genetics $d, h$ and $m$ are used in the same way as in the earlier paper (see fig. 1 in Mather 1987) with $m$ and $h$ taking sign and measured in units of $d$.

The same basic assumptions are also made as before, notably

(i) that mating is at random in the population;

(ii) that the frequency distribution of the phenotypic expression of the primary character is normal and is undisturbed by both nonallelic interaction and the consequences of linkage;

(iii) that the fitness is also given by a normal distribution; and

(iv) that the differences in average expression due to allelic substitution at any one locus are sufficiently small by comparison with the total phenotypic variation to render the loss of 
Table 1 The effect of stabilising selection on locus $A$, a when a second polymorphic locus $\mathrm{B}, \mathrm{b}$ is also involved

$$
\begin{aligned}
& w_{\mathrm{AA}}=1-\theta d_{\mathrm{a}}^{2}\left(m_{\mathrm{a}}+1\right)^{2} \quad-2 \theta d_{\mathrm{a}}\left(m_{\mathrm{a}}+1\right) d_{\mathrm{b}} \bar{x}_{\mathrm{b}} \quad-\theta d_{\mathrm{b}}^{2} T_{\mathrm{b}} \\
& w_{\mathrm{Aa}}=1-\theta d_{\mathrm{a}}^{2}\left(m_{\mathrm{a}}+h_{\mathrm{a}}\right)^{2} \quad-2 \theta d_{\mathrm{a}}\left(m_{\mathrm{a}}+h_{\mathrm{a}}\right) d_{\mathrm{b}} \bar{x}_{\mathrm{b}} \quad-\theta d_{\mathrm{b}}^{2} T_{\mathrm{b}} \\
& w_{\mathrm{aa}}=1-\theta d_{\mathrm{a}}^{2}\left(m_{\mathrm{a}}-1\right)^{2} \quad-2 \theta d_{\mathrm{a}}\left(m_{\mathrm{a}}-1\right) d_{\mathrm{b}} \bar{x}_{\mathrm{b}} \quad-\theta d_{\mathrm{b}}^{2} T_{\mathrm{b}} \\
& w_{\mathrm{A}}=1-\theta d_{\mathrm{a}}^{2}\left[u_{\mathrm{a}}\left(m_{\mathrm{a}}+1\right)^{2}+v_{\mathrm{a}}\left(m_{\mathrm{a}}+h_{\mathrm{a}}\right)^{2}-2 \theta d_{\mathrm{a}}\left(m_{\mathrm{a}}+u_{\mathrm{a}}+v_{\mathrm{a}} h_{\mathrm{a}}\right) d_{b} \bar{x}_{\mathrm{b}}-\theta d_{\mathrm{b}}^{2} T_{\mathrm{b}}\right. \\
& \begin{array}{lll}
\bar{w}=1-\theta d_{\mathrm{a}}^{2} T_{\mathrm{a}} & -2 \theta d_{\mathrm{a}} \bar{x}_{\mathrm{a}} d_{\mathrm{b}} \bar{x}_{\mathrm{b}} \quad-\theta d_{\mathrm{b}}^{2} T_{\mathrm{b}}
\end{array}
\end{aligned}
$$

$\Delta u_{\mathrm{a}}=\frac{\theta}{\bar{x}} u_{\mathrm{a}} v_{\mathrm{a}}\left\{d_{\mathrm{a}}^{2}\left[v_{\mathrm{a}}-u_{\mathrm{a}}\left(1-h_{\mathrm{a}}-2 m_{\mathrm{a}} h_{\mathrm{a}}\right)-2 m_{\mathrm{a}}\right]-2 d_{\mathrm{a}}\left[1+\left(v_{\mathrm{a}}-u_{\mathrm{a}}\right) h_{\mathrm{a}}\right] d_{\mathrm{b}} \overline{\mathrm{x}}_{\mathrm{b}}\right\}$

Note: $\bar{x}=[m+(u-v)+2 u v h]$ and $T=\left[u^{2}(m+1)^{2}+2 u v(m+h)^{2}+v^{2}(m-1)^{2}\right]$.

fitness entailed by the departures of the values of the three genotypes from the optimum expression, to be satisfactorily approximatable by the squares of these departures.

One further point must be made. It will be seen that the factor $\lambda / 2$ no longer appears in the common term of the expression representing the effects of stabilising selection which, following Kimura (1981), was used in the earlier publication. This is because I have come to doubt the applicability of the Taylor series, by which Kimura separates the effects of directional and stabilising selection, to the consequences of natural or quasi-natural selection as opposed to artificial selection. Under natural selection the key cause of both change and stability lies in the differences in reduction of fitness shown by individuals differing in their departures from the optimum in respect of the primary character; and this will apply to the directional component in the selection as well as the stabilising one.

The factor $\lambda / 2$ has been replaced by $\theta$ which, like $\lambda / 2$, will allow for the varying values of the coefficient-in the fitness function, but which does not carry implications beyond this. It is used with the squares of the deviation of the primary character from the optimum. It should be added that the change to $\theta$ makes no difference to the comparative conclusions reached in the earlier, as well as the present, communication.

\section{TWO LOCI}

In the earlier paper it was pointed out that although the genes at the relevant loci are assumed not to show non-allelic interaction (epistasis) in respect of the primary character, the reduction in fitness due to deviation from the optimum phenotype may well do so. For example, an individual of genotype AABB will deviate phenotypically from the optimum by $d_{\mathrm{a}}\left(m_{\mathrm{a}}+1\right)+\left(d_{\mathrm{b}}\left(m_{\mathrm{b}}+1\right)\right.$. The reduction in fitness, however, will be proportional to $\left[d_{\mathrm{a}}\left(m_{\mathrm{a}}+1\right)+d_{\mathrm{b}}\left(m_{\mathrm{b}}+1\right)\right]^{2}$, which can be rewritten as $\left[d_{\mathrm{a}}\left(m_{\mathrm{a}}+1\right)\right]^{2}+2\left[d_{\mathrm{a}}\left(m_{\mathrm{a}}+1\right)\right] \times$ $\left[d_{\mathrm{b}}\left(m_{\mathrm{b}}+1\right)\right]+\left[d_{\mathrm{b}}\left(m_{\mathrm{b}}+1\right)\right]^{2}$, the second term arising from the non-allelic interaction between the two loci. If we similarly partition the reduction in fitness of $\mathrm{AABb}$ and $\mathrm{AAbb}$ individuals and then average over the three $B, b$ genotypes we obtain the reduced fitness shown in the first line of table 1 .

The second and third lines of table 1 set out the corresponding partitions for $\mathrm{Aa}$ and aa respectively, and the fifth line gives the average fitness $(\bar{w})$ of the nine genotypes. The same value is, of course, obtained for $(\bar{w})$ if we start by separating the three B,b genotypes average over A,a. The difference in the two approaches is that the one used enables us to investigate the selection pressure on $\mathrm{A}$, a, while the other would yield corresponding information about $\mathrm{B}, \mathrm{b}$.

Returning to table 1 , and again following Kimura (1981), the value of $w_{\mathrm{A}}$ can be obtained as $u_{\mathrm{a}} w_{\mathrm{AA}}+v_{\mathrm{a}} w_{\mathrm{Aa}}$ and from it $\Delta u_{\mathrm{a}}=u_{\mathrm{a}}\left(w_{\mathrm{A}}-\bar{w}\right) / \bar{w}$, which is shown in the final line of the table. It will be noted that in each line of the table the third term, which represents the main contribution of $\mathrm{B}, \mathrm{b}$ to the loss of fitness, is the same for all three A,a genotypes and so cancels out during the calculation of $\Delta u_{\mathrm{a}}$. The second term, which represents the interaction between the two loci, vanishes only when $\quad d_{\mathrm{b}} \bar{x}_{\mathrm{b}}=d_{\mathrm{b}}\left(m_{\mathrm{b}}+u_{\mathrm{b}}-v_{\mathrm{b}}+2 u_{\mathrm{b}} v_{\mathrm{b}} h_{\mathrm{b}}\right)=0$, as already found earlier (Mather 1987), or when $d_{\mathrm{a}}\left[1+\left(v_{\mathrm{a}}-u_{\mathrm{a}}\right) h_{\mathrm{a}}\right]=0 .{ }^{*}$ It is of interest to note that

* In the earlier paper these were shown incorrectly as

$$
d_{\mathrm{a}}\left[1+\left(u_{\mathrm{a}}-v_{\mathrm{a}}\right) h_{\mathrm{a}}\right]=0 \text { and } \quad D_{\mathrm{R}}=S d^{2}\left[1+(v-u) h^{2}\right]
$$


not only is the former the mean expression of the phenotype in respect of $B, b$, but the latter is the basis of the contribution of $A$, a to the additive genotypic variation of the population,

$$
D_{\mathrm{R}}=S\left\{4 u v d^{2}[1+(v-u) h]^{2}\right){ }^{*}
$$

The expression for $\Delta u_{\mathrm{a}}$ as derived in table 1 can be re-written as

$$
\begin{aligned}
\Delta u_{\mathrm{a}}=\frac{\theta}{\bar{w}} u_{\mathrm{a}} v_{\mathrm{a}}\{ & d_{\mathrm{a}}^{2}\left[\left(v_{\mathrm{a}}-u_{\mathrm{a}}\right)\right. \\
& \times\left(1-h_{\mathrm{a}}^{2}-2 h_{\mathrm{a}}\left(m_{\mathrm{a}}+d_{\mathrm{b}} \bar{x}_{\mathrm{b}} / d_{\mathrm{a}}\right)\right) \\
& \left.\left.-2\left(m_{\mathrm{a}}+d_{\mathrm{b}} \bar{x}_{\mathrm{b}} / d_{\mathrm{a}}\right)\right]\right\}
\end{aligned}
$$

which at equilibrium equals 0 . The equilibrium value of $v_{\mathrm{a}}-u_{\mathrm{a}}$ is thus

$$
2 m_{\mathrm{a}}^{\prime} /\left(1-h_{\mathrm{a}}^{2}-2 m_{\mathrm{a}}^{\prime} h_{\mathrm{a}}\right)
$$

where $m_{\mathrm{a}}^{\prime}=m_{\mathrm{a}}+r_{\mathrm{b} / \mathrm{a}} \bar{x}_{\mathrm{b}}$ and $r_{\mathrm{b} / \mathrm{a}}=d_{\mathrm{b}} / d_{\mathrm{a}}$. Thus the expressions derived in the earlier paper concerning the range over which A, a will show a stable polymorphism, so conserving variation, as opposed to fixation of either A or a, will still be valid despite the non-allelic interaction, provided that $m_{\mathrm{a}}$ is replaced by $m_{\mathrm{a}}^{\prime}$ as just defined. Where polymorphism occurs, the contribution of locus $A$,a to the mean expression of the character at equilibrium is

$$
d_{\mathrm{a}} \bar{x}_{\mathrm{a}}^{\prime}=d_{\mathrm{a}}\left[m_{\mathrm{a}}^{\prime}+\left(\hat{u}_{\mathrm{a}}-\hat{v}_{\mathrm{a}}\right)+2 \hat{u}_{\mathrm{a}} \hat{v}_{\mathrm{a}} h_{\mathrm{a}}\right] .
$$

The corresponding analysis can be carried out for $\mathrm{B}, \mathrm{b}$ and it shows similarly that at equilibrium

$$
\Delta_{\mathrm{b}}=\left\{u_{\mathrm{b}} v_{\mathrm{b}} d_{\mathrm{b}}^{2}\left[\left(v_{\mathrm{b}}-u_{\mathrm{b}}\right)\left(1-h_{\mathrm{b}}^{2}-2 m_{\mathrm{b}}^{\prime} h_{\mathrm{b}}\right)-2 m_{\mathrm{b}}^{\prime}\right]\right\}=0
$$

where $m_{\mathrm{b}}^{\prime}=m_{\mathrm{b}}+r_{\mathrm{a} / \mathrm{b}} \bar{x}_{\mathrm{a}}$.

The contribution of $\mathrm{B}, \mathrm{b}$ to the mean expression of the character will then be

$$
d_{\mathrm{b}} x_{\mathrm{b}}=d_{\mathrm{b}}\left[m_{\mathrm{b}}^{\prime}+\left(\hat{u}_{\mathrm{b}}-\hat{v}_{\mathrm{b}}\right)+2 \hat{u}_{\mathrm{b}} \hat{v}_{\mathrm{b}} h_{\mathrm{b}}\right]
$$

Thus stable polymorphism at each of the loci depends on the mean expression of the other.

\section{ANY NUMBER OF LOCI}

The approach used above for two loci can be generalised to deal with $k$ loci. Again, assuming the absence of non-allelic interaction in the determination of the primary phenotype, the expression of an individual homozygous for the increasing allele at all loci will deviate from the optimum by

$$
\left[d_{\mathrm{a}}\left(m_{\mathrm{a}}+1\right)+d_{\mathrm{b}}\left(m_{\mathrm{b}}+1\right) \cdots+d_{\mathrm{k}}\left(m_{\mathrm{k}}+1\right)\right]
$$

and the ensuing reduction in fitness will then be proportional to the square of this expression. Corresponding expressions can be obtained for the other genotypes which are distinguished by being AA. Then, averaging over all these AA genotypes, the reduction in fitness will be proportional to

$$
d_{\mathrm{a}}^{2}\left[\left(m_{\mathrm{a}}+1\right)+2\left(m_{\mathrm{a}}+1\right) S_{\mathrm{b}}^{\mathrm{k}} x_{\mathrm{i}} r_{\mathrm{i} / \mathrm{a}}+T\right]
$$

where $T$ is now a complex expression which includes both the interactions as well as the main effects of loci $\mathrm{B}, \mathrm{b}-K, k$ but does not include any item involving locus $A$,a and so will vanish when the effects of selection on A,a are being assessed, just as the item in $T$ vanished in table 1 . This then provides the generalised form of line 1 in table 1 . Similar treatment of the genotypes distinguished by being $\mathrm{Aa}$, and then those distinguished by being aa, give the counterparts of lines 2 and 3 in the table.

Proceeding to find $\Delta_{\mathrm{a}}$ as in table 1 yields

$$
\begin{gathered}
\Delta_{\mathrm{a}}=\frac{\theta}{\bar{w}} u_{\mathrm{a}} v_{\mathrm{a}} d_{\mathrm{a}}^{2}\{[ \\
\left.\left.v_{\mathrm{a}}-u_{\mathrm{a}}\right)\left(1-h_{\mathrm{a}}^{2}-2 m_{\mathrm{a}} h_{\mathrm{a}}\right)-2 m_{\mathrm{a}}\right] \\
\left.-2\left[1+\left(v_{\mathrm{a}}-u_{\mathrm{a}}\right) h_{\mathrm{a}}\right] S_{\mathrm{b}}^{\mathrm{k}} \overline{\mathrm{x}}_{\mathrm{i}} r_{\mathrm{i} / \mathrm{a}}\right\}
\end{gathered}
$$

Equating this to 0 gives $\hat{v}_{\mathrm{a}}-\hat{u}_{\mathrm{a}}=$ $2 m_{\mathrm{a}}^{\prime} /\left(1-h_{\mathrm{a}}^{2}-2 m_{\mathrm{a}}^{\prime} h_{\mathrm{a}}\right)$ as before but now with $m_{\mathrm{a}}^{\prime}=$ $m_{\mathrm{a}}+S_{\mathrm{b}}^{\mathrm{k}} \overline{\mathrm{x}}_{\mathrm{i}} r_{\mathrm{i} / \mathrm{a}}$, i.e. involving all $k$ loci in the polygenic system mediating the heritable variation of the primary character in question. Correspondingly, $d_{\mathrm{a}} x_{\mathrm{a}}^{\prime}=d_{\mathrm{a}}\left(m_{\mathrm{a}}^{\prime}+\hat{u}_{\mathrm{a}}-\hat{v}_{\mathrm{a}}+2 \hat{u}_{\mathrm{a}} \hat{v}_{\mathrm{a}} h_{\mathrm{a}}\right)$. So it too depends on all the loci in the system. $k$ is likely to be some 20 or more each for sternopleural and abdominal chaeta number in Drosophila melanogaster (see Mather, 1983; Shrimpton and Robertson, 1988a, b). Falconer (1971) has estimated that some 80 loci were involved in the determination of litter size in his population of mice; and "Student" (1934) even expressed the opinion that the number of loci involved in the determination of protein and oil content in the Illinois Experimental Station selection experiment with maize was likely to be nearer to 200 than 20 .

Much smaller numbers of QTLs (quantitative trait loci) have been found by Patterson et al. (1988) to be involved in differences between a commercial variety of tomato and a wild relative. The aim of this work, however, was evidently not to obtain an estimate of the number of loci involved in a given polygenic system, but to find a means of identifying QTLs which could be useful in plant breeding. Its experimental approach and the statistical assessment of the data were also quite different from those of the earlier experiments 
quoted above. An average of only five loci was found for three commercially important characters. The authors recognise that these numbers must be minimal and, indeed, their criteria and analyses may well be responsible for their low estimates, which will not be considered further in our present context.

Returning to our earlier estimates, no matter whether $k=20,80$ or 200 the implication of our present finding is that the effect of stabilising selection on the allele frequencies at any one locus will depend also on both the overall situation at the other loci in the system and the concommitant effects of selection at all these other loci. Given that $k$ may well be 20 or more, and that both $m$ and $h$ can take sign, we must expect that $\bar{x}_{\mathrm{i}} r_{\mathrm{i} / \mathrm{a}}$ can, and indeed will, vary in sign from locus to locus and so tend to cancel out one another's effects.

We will return later to the consequences of this interdependence, but first we must look at the way in which $m_{\mathrm{a}}, h_{\mathrm{a}}$ and $S_{\mathrm{b}}^{k} \bar{x}_{\mathrm{i}} r_{\mathrm{i} / \mathrm{a}}$ combine to produce their joint equilibrium value of $\hat{v}_{\mathrm{a}}-\hat{u}_{\mathrm{a}}$, starting with the interplay of $m_{\mathrm{a}}$ and $h_{\mathrm{a}}$. Confining ourselves for a moment to the single locus, allele a will become fixed when $\hat{v}_{\mathrm{a}}-\hat{u}_{\mathrm{a}}=$ $2 m_{\mathrm{a}} /\left(1-h_{\mathrm{a}}^{2}-2 m_{\mathrm{a}} h_{\mathrm{a}}\right)=1$, that is when $\left(1+h_{\mathrm{a}}\right) \times$ $\left(1-h_{\mathrm{a}}-2 m_{\mathrm{a}} h_{\mathrm{a}}\right)=0$. Thus fixation occurs when $h_{\mathrm{a}}=-1$ or $1-2 m_{\mathrm{a}}$, with polymorphism appearing when $h_{\mathrm{a}}$ lies between these values. Similarly A becomes fixed when $v_{\mathrm{a}}-u_{\mathrm{a}}=-1$ and $h_{\mathrm{a}}=1$ or $-\left(1-2 m_{\mathrm{a}}\right)$, again with polymorphism at intermediate values. $m$ is plotted against $h$ in fig. 1 which shows that the range of $h$ over which $1>(v-u)>$ -1 changes with $m$, being greatest when $m=0$ and vanishing when $m= \pm 1$. Furthermore for any given value of $m$, the value of $|v-u|$ is at a minimum when $h=-m$. The value of $h$ will have been determined by the mutation which initiated the second allele at the locus in question, and there is no reason to expect it to depend on the value of $m$. So where $m$ is positive, the greater its value the smaller the effective range of $h$ and the greater the chance of fixation of allele a, and even where polymorphism supervenes, the greater the value of $v$. Similarly, with a negative $m$ the greater correspondingly will be the chance of fixation of allele $\mathrm{A}$, and the greater the value of $u$ where polymorphism supervenes.

So, returning to $m^{\prime}$, if $m_{\mathrm{a}}$ and $S_{\mathrm{b}}^{k} \bar{x}_{\mathrm{i}} r_{\mathrm{i} / \mathrm{a}}$ are both positive their combined effect will be to increase the frequency of allele a, and indeed to fix it if $S_{\mathrm{b}}^{k}{ }^{-} x_{\mathrm{i}} r_{\mathrm{i} / \mathrm{r}}$ is large enough, a being of course the decreasing allele. Thus where loci $\mathrm{B}, \mathrm{b}-K, k$ have an overall effect of increasing the mean expression of the primary character they increase the

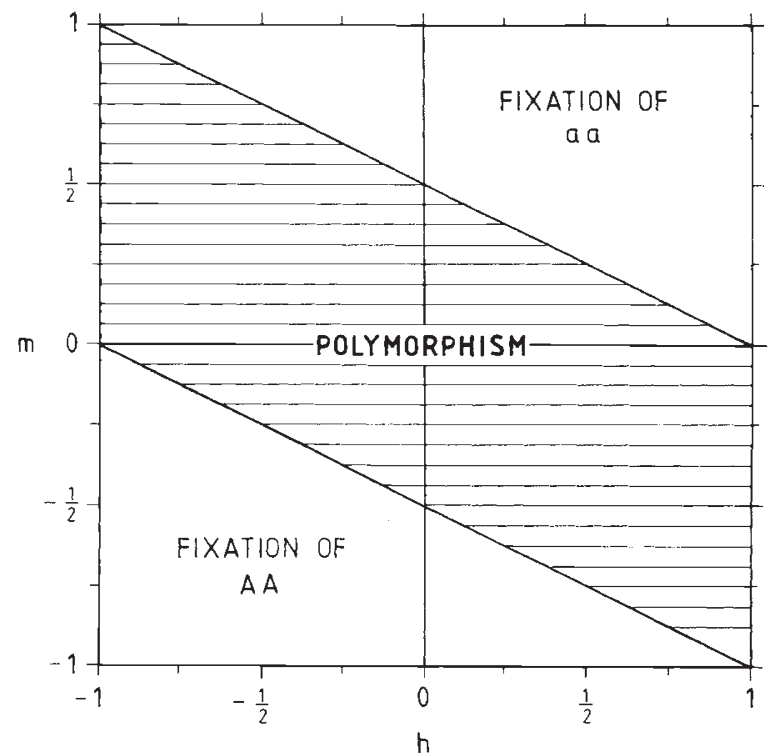

Figure 1 The relation between $m$ and $h$ in the determination of polymorphism by stabilising selection. Polymorphism arises more frequently when $m$ and $h$ are of opposite sign. Fixation of allele $a$ is the alternative to polymorphism when $m$ is positive, and fixation of allele $A$ when $m$ is negative.

frequency, even to the point of fixation of the decreasing allele at locus, Aa. Correspondingly, where $m_{\mathrm{a}}$ and $S_{\mathrm{b}}^{k} \bar{x}_{\mathrm{i}} r_{\mathrm{i} / \mathrm{a}}$ are both negative the consequence will be an increase in frequency, even to the point of fixation, of $\mathrm{A}$, the increasing allele at the A,a locus.

Where $m_{\mathrm{a}}$ and $S_{\mathrm{b}}^{k} \bar{x}_{\mathrm{i}} r_{\mathrm{i} / \mathrm{a}}$ are opposite in sign, $m^{\prime}$ will lie between them and so will be closer than $m_{\mathrm{a}}$ to 0 . Since the optimum is the origin for measuring $m$, this implies an approach to the optimal expression of the primary character, as well as potentially increasing the conserved variation.

No matter, however, whether A,a is moved towards fixation of one allele or towards a reinforced polymorphism, the inclusion of $S_{\mathrm{b}}^{k} \bar{x}_{\mathrm{i}} r_{\mathrm{i} / \mathrm{a}}$ in $m_{\mathrm{a}}^{\prime}$ ensures that at equilibrium the allele frequencies at locus $\mathrm{A}$, a will become adjusted to take into account the allele frequencies and other features at all the remaining loci. Since the same stabilising selection will also have been acting on all these other loci, when stability is attained the properties of each of them will have become adjusted to take into account those of locus A,a and indeed of all the other loci too. The net results of the selection will thus be that at stability the overall expression of the primary character will be $S_{\mathrm{b}}^{k} d_{\mathrm{i}} x_{\mathrm{i}}$, which is of course the mean expression of the primary character, and since the polymorphism at every 
locus is stable, this mean expression will not change save by sampling variation, provided that the environment does not produce a change in the optimum expression.

This stability does not, however, imply that the mean is necessarily at the optimum. It may well be there, as a departure of the mean from the optimum implies a loss of fitness, and provided that the total variation in the system is adequate to fuel the change, as indeed must commonly be the case, selection will push the mean to the optimum. In any case, even if the mean were not at the optimum, further mutation may occur at one or more of the $k$ loci we have been discussing, or at other loci which affect the character in question but which hitherto have been mono-allelic, or new alleles may be introduced by migration from another population. No matter whether the introduction of any such new allele changed the value of $m^{\prime}$ at one of the existing polymorphic loci, or introduced a new $m^{\prime}$ at a previously monomorphic locus, the whole system would react to the change. Given that the new allele survived the vagaries of sampling variation and that it moved the re-adjusted $S_{\mathrm{a}}^{k} \bar{d}_{\mathrm{i}} \bar{x}_{\mathrm{i}}$ nearer to the optimum it would confer an advantage on the whole system and would be maintained by selection either as a component of a polymorphism or by its own fixation if the advantage confered were sufficiently large. Thus, although the optimum expression of the character must itself show some fluctuation from generation to generation, or season to season, provided that this fluctuation was not too great we would expect the mean of the primary character to move towards the optimum until the two were regularly in close proximity even if not actually equal.

\section{EXPERIMENTAL EVIDENCE}

In 1970 Kearsey and Barnes showed that in the Texas cage population of Drosophila melanogaster the fitnesses of individuals are related to their numbers of sternopleural chaetae, their capacity for survival under crowded conditions being greatest when the chaetae numbers are near to the mean and declining as the number departs increasingly from it, no matter in which direction. Thus the mean chaeta number must be at or near the optimum. It was further shown by Linney et al. (1971) that these differences in survival rate had nothing to do with the level of heterozygosity of the flies, as they were also observed when the individuals of differing chaeta numbers were all from inbred lines: the loss of fitness must be due to the departure from the mean of the chaeta numbers themselves, which reinforces the conclusion that the mean must be at or near the optimal expression.

The average numbers of chaetae were later ascertained for each of eighteen inbred lines derived from this population, and although the lines differed among themselves the overall average of the eighteen did not differ significantly from the mean chaeta number of a sample of the population itself when raised under corresponding conditions (Caligari and Mather, 1980).

In a randomly breeding population the mean contribution of locus $\mathrm{A}, \mathrm{a}$ to the mean expression of the character is $d_{\mathrm{a}}\left(m_{\mathrm{a}}+u_{\mathrm{a}}-v_{a}+2 u_{\mathrm{a}} v_{\mathrm{a}} h_{\mathrm{a}}\right)$, which, we may observe, will also cover loci at which one allele has been fixed by earlier selection. An inbred line derived from the population is thus expected to have a mean expression of $d_{\mathrm{a}}\left(m_{\mathrm{a}}+1\right)$ in $u_{\mathrm{a}}$ of cases and of $d_{\mathrm{a}}\left(m_{\mathrm{a}}-1\right)$ in $v_{\mathrm{a}}$ of cases. Equally, if $m_{\mathrm{a}}$ is negative the expectations are $-d_{\mathrm{a}}\left(m_{\mathrm{a}}+1\right)$ in $u_{\mathrm{a}}$ of cases and $-d_{\mathrm{a}}\left(m_{\mathrm{a}}-1\right)$ in $v_{\mathrm{a}}$ of cases. The overall mean of a fair sample of inbreds from the population should thus have a mean of $S_{\mathrm{a}}^{k} d_{\mathrm{i}}\left(m_{i}+u_{\mathrm{i}}-v_{\mathrm{i}}\right)$ where $m, u$ and $v$ all take sign, $u$ being always of the same sign as $m$, and $\mathrm{v}$ being of opposite sign. Thus both $S_{\mathrm{a}}^{k} d_{\mathrm{i}}\left(m_{i}+\right.$ $\left.u_{i}-v_{\mathrm{i}}\right)$ and $S_{\mathrm{a}}^{k} d_{\mathrm{i}} x_{\mathrm{i}}$ are at or near the optimum in the present case.

Two conclusions follow. In the first place, in view of the differences among the eighteen inbreds, $S_{\mathrm{a}}^{k} d_{\mathrm{i}}\left(m_{\mathrm{i}}+u_{\mathrm{i}}-v_{i}\right) \simeq 0$ must imply an equality or near equality of the summed action of loci with positive $m$ and those of negative $m$. Secondly, since both the population mean and the mean of the inbreds are at or near the optimum $S_{\mathrm{a}}^{k} 2 d_{\mathrm{i}} u_{\mathrm{i}} v_{\mathrm{i}} h_{\mathrm{i}} \simeq 0$. This requires that either all $h \simeq 0$ or that the consequences of positive and negative $h$ balance one another. It is known, however, that $h \neq 0$ (Barnes and Kearsey, 1970). So not only must $h$ vary in sign, but it must, do so within restrictions imposed by the limits on the combinations in value of $h$ and $m$ that are consonant with polymorphisms at the loci; and since $S_{\mathrm{a}}^{k} d_{\mathrm{i}}\left(m_{\mathrm{i}}+u_{\mathrm{i}}-v_{\mathrm{i}}\right)=0$ so correspondingly must $S_{\mathrm{a}}^{k} 2 d_{\mathrm{i}} u_{\mathrm{i}} v_{\mathrm{i}} h_{\mathrm{i}}$. This surely implies the action of stabilising selection.

These conclusions depend on comparisons in respect of fitness of the means of both the population itself and the inbred lines derived from it, the distribution of fitness having been related to chaeta number in a separate and quite demanding experiment. This gives a direct and powerful approach to the understanding of the selection at work, but such a detailed knowledge of the relation between 
fitness and expression of the primary character may not always be an essential requirement. Since at any locus showing stable polymorphism the heterozygote must be fitter, and therefore phenotypically nearer to the optimum than both homozygotes, the fittest phenotype will be that in which all polymorphic loci are represented by heterozygotes. Furthermore, as we have seen, $m$ and $h$ both take sign and both are expected to be balanced round the optimum. So given that the frequency distribution of the primary character is symmetrical, the fittest (i.e. the optimal) phenotype should be at the mean of the phenotypic distribution, and also be the one where all the polymorphic loci are heterozygous. In this sense it will be the phenotype of maximum dominance, $S_{\mathrm{a}}^{k} 2 d_{\mathrm{i}} u_{\mathrm{i}} v_{\mathrm{i}} h_{\mathrm{i}}$, though it may equally be described as the phenotype of balanced dominance since $S_{\mathrm{a}}^{k} 2 d_{\mathrm{i}} u_{\mathrm{i}} v_{\mathrm{i}} h_{\mathrm{i}}$ will $=0$.

This relationship has been used by Caligari and Mather (1980) and Caligari (1981) to locate the optimum phenotype in terms of the frequency distribution of the primary character, and compare it with the mean of the distribution. They used data from half-diallel crosses among inbred lines derived from the Texas cage population in two experiments described and utilised by Mather (1987) in a different connection. Five characters were scored: the number of sternopleural chaetae $(M)$; the sensitivity of the chaeta number to temperature change $(S)$; and the number of coxal chaetae on the three pairs of legs, presented in terms of the three classes of genes, $\alpha, \beta$ and $\gamma$, postulated by Mather and Hanks (1978) to account for differences among the chaeta numbers between them. The differences between the mean scores of the parental flies and the estimated phenotypes of maximal dominance (i.e., optima) are set out in table 2 , from which it will be seen that in only one case, that of the $\alpha$ genes for coxal chaetae, is there a significant (though still small) difference between the mean of the parents and the optimum $(P=$ $0 \cdot 05-0 \cdot 01$ ).

Even this may be accountable. Kearsey and Barnes (loc. cit.) found that although the relative fitnesses of individuals when plotted against their number of sternopleural chaetae fell away on both sides of the optimal value, they did not do so in a symmetrical fashion, with the consequence that the mean chaeta number must exceed the optimum. They showed, however, that a straightforward transformation of the chaeta number could result in a symmetrical distribution where mean and optimum would coincide. The $\alpha$ coxal genes in Caligari's experiment could be in a similar case, albeit one in which the deviations of the mean from the optimum was negative. Unlike Kearsey and Barnes, Caligari found no significant difference between mean and optimum for sternopleurals; but this was a smaller experiment using a completely different technique, and a mere difference in the environment under which the flies were raised could change the optimum as well as the mean and hence also the departure of one from the other. Clearly, the relation between mean and optimum under stabilising selection is worthy of further investigation using appropriately designed experiments; but such experimental data as we have at present agrees sufficiently well with our theoretical expectations.

\section{CONCLUSIONS}

Like other nuclear genes, the members of polygenic systems are carried on the chromosomes. They segregate, recombine and can show dominance. They also mutate from time to time, as other genes do. They differ, however, from the more familiar type of gene in the way that they express themselves

Table 2 Comparison of mean with phenotype of maximum dominance for five characters in Drosophila melanogaster. Data from Caligari and Mather (C and M) and Caligari (C) relating to sternopleural chaeta number $(M)$ and its temperature sensitivity $(S)$, and to coxal chaeta numbers attributable to the $\alpha, \beta$ and $\gamma$ types of gene

\begin{tabular}{|c|c|c|c|c|c|c|}
\hline \multirow{2}{*}{$\begin{array}{l}\text { Source of data } \\
\text { Character }\end{array}$} & \multicolumn{3}{|l|}{$C \& M$} & \multicolumn{3}{|l|}{$\mathrm{C}$} \\
\hline & M & $\mathrm{S}$ & M & $\alpha$ & $\beta$ & $\gamma$ \\
\hline Maximum dominance phenotype & $19 \cdot 21$ & $0 \cdot 92$ & $17 \cdot 70$ & $15 \cdot 80$ & $5 \cdot 68$ & $4 \cdot 09$ \\
\hline Mean of parents & $19 \cdot 15$ & $0 \cdot 45$ & $18 \cdot 48$ & $15 \cdot 18$ & $6 \cdot 01$ & $3 \cdot 36$ \\
\hline $\begin{array}{l}\text { Difference and } \\
\text { its standard error }\end{array}$ & $\begin{array}{r}0.06 \\
\pm 0.78\end{array}$ & $\begin{array}{r}0 \cdot 47 \\
\pm 0 \cdot 24\end{array}$ & $\begin{array}{l}-0 \cdot 78 \\
\pm 0 \cdot 75\end{array}$ & $\begin{aligned} & 0 \cdot 62^{*} \\
\pm & 0 \cdot 28\end{aligned}$ & $\begin{array}{l}-0 \cdot 33 \\
\pm 0 \cdot 39\end{array}$ & $\begin{array}{r}0.73 \\
\pm 0.45\end{array}$ \\
\hline
\end{tabular}

\footnotetext{
* Probability $0 \cdot 05-0 \cdot 01$
} 
phenotypically: the individual members of a polygenic system have relatively small effects, which are similar to those of each other and capable of, on the one hand, supplementing and, on the other, balancing one another's effects. This interchangeability at the phenotypic level makes them interdependent in their response to selection. As we have seen, the response of each member gene to stabilising selection is affected by every other gene in the system and in its turn affects the response of all other genes.

This interdependence is a basic feature of the system. Each gene-pair is marked by its values of three parameters: $d$, which is half the difference, in respect of the primary character, between the two homozygotes, aa and AA; $h$ which is the difference in effect between $\mathrm{Aa}$ and the mid-parent between aa and AA; and $m$ which is the departure of the mid-parent from $\mathrm{O}$, the optimum expression of the primary character. Now, the values of $d$ and $h$ must have been fixed by the mutation which gave rise to the gene-pair, and so represent fixed differences. At the same time the overall value of $S_{\mathrm{a}}^{k} m_{\mathrm{i}}$, marking as it does the difference in phenotype between the overall mean expression of all the genes in the system, on the one hand, and the optimum on the other, will be liable to variation as a result of both genetic change anywhere in the polygenic system and environmental alteration expressed in the optimum, as indeed is reflected in the composition of the parameter $m^{\prime}$ that we have been led to define.

Yet, although in principle the values of $d$ and $h$ shown by a mutant gene-pair will depend neither on the other genes in the system nor on the stability of the optimum, the fate of that gene-pair may well depend on both. Unless the mutation by which it originates is in the right direction and of large enough effect for the mutant homozygote to be on the opposite side of the optimum from its precursor (i.e. of a size dependent on the value of $m$ ) it cannot result in a polymorphism and so cannot contribute to the pool of conserved variation. Also as we observed earlier (see fig. 1) the value of $m$ sets limits to the range of $h$ over which the genepair can show polymorphism, and the value of $m$ can be modified by change of both optimum and the behavior of the other genes in the system. Thus the values of $d, h$ and $m$ of a gene-pair must be consonant not only with each other but also with those of the other gene-pairs in the system if it is to contribute to polymorphism and the variation of the population. Failing such consonance, one or other allele will go to fixation. Such fixation may well be helpful to the fitness of the population at the time, but it will be a one-off gain to the genotype; unless and until further mutation occurs at such a locus it cannot contribute further to the lability of the system and its capacity for continuing adaptation.

Acknowledgements I am grateful to Drs M. J. Lawrence and M. J. Kearsey for their comments on an early version of this script and to Dr Lawrence also for his help in the preparation of the manuscript for the printer.

\section{REFERENCES}

BARNES, B. W. AND KEARSEY, M. J. 1970. Variation for metrical characters in Drosophila populations. I. Genetic analysis. Heredity, 25, 1-10.

CALIGARI, P. D. S. 1981. The selectively optimal phenotypes of the coxal chaetae in Drosophila melanogaster. Heredity, 47, $79-85$.

CALIGARI, P. D. S. AND MATHER, K. 1980. Dominance, allele frequency and selection in a population of Drosophila melanogaster. Proc. R. Soc. Lond. B, 208, 163-187.

FALCONER, D. S. 1971. Improvement of litter size in a strain of mice at a selection limit. Genetic Res., 17, 215-235.

KEARSEY, M. J. AND BARNES, B. W. 1970. Variation for metrical characters in Drosophila populations. II. Natural selection. Heredity, 25, 11-21.

KIMURA, M. 1981. Possibility of extensive neutral evolution under stabilising selection with special reference to nonrandom usage of synonymous codons. Proc. Natl Acad. Sci. USA, 78, 5773-5777.

LINNEY, R., BARNES, B. W. AND KEARSEY, M. J. 1971. Variation for metrical characters in $D$ rosophila populations. III. The nature of selection. Heredity, 27, 163-174.

MATHER, K. 1983. Response to selection. In: Ashburner, M., Carson, H. L. and Thompson, J. N. Jr. (eds) Genetics and Biology of Drosophila 30, Academic Press, London, pp. 155-221.

MATHER, K. 1987. Consequences of stabilising selection for polygenic variation. Heredity, 58, 267-277.

MATHER, K. AND HANKS, M. J. 1978. Genetics of coxal chaetae in Drosophila melanogaster. I. Variation in gene action. Heredity, 40, 71-96.

PATTERSON, A. H., LANDER, E. S., HEWITT, J. D., PETERSON, S., LINCOLN, S. E. AND TANKSLEY, S. D. 1988. Resolution of quantitative traits into Mendelian factors by using a complete RFLP linkage map. Nature, 335, 721-726.

SHRIMPTON, A. E. AND ROBERTSON, A. 1988a. The isolation of polygenic factors controlling bristle score in Drosophila melanogaster. I. Allocation of third chromosome bristle effects to chromosome sections. Genetics, 18, 437-443.

SHRIMPTON, A. E. AND ROBERTSON, A. 1988b. The isolation of polygenic factors controlling bristle score in Drosophila melanogaster. II. Distribution of third chromosome bristle effects within chromosome sections. Genetics, 18, 445-459.

"STUDENT". 1934. A calculation of the minimum number of genes in Winter's selection experiment. Ann. Eugenics, 6, $77-82$. 